\title{
Tomographic Particle Image Velocimetry and Dynamic Mode Decomposition (DMD) in a Rectangular Impinging Jet: Vortex Dynamics and Acoustic Generation
}

\author{
Hassan H. Assoum ${ }^{1}$, Jana Hamdi ${ }^{2} \mathbb{D}$, Marwan Alkheir ${ }^{3}$, Kamel Abed Meraim ${ }^{3}$, Anas Sakout ${ }^{3} \mathbb{D}$, Bachar Obeid $^{4}$ \\ and Mouhammad El Hassan $5, *$ (D)
}

check for updates

Citation: Assoum, H.H.; Hamdi, J.; Alkheir, M.; Abed Meraim, K.; Sakout,

A.; Obeid, B.; El Hassan, M.

Tomographic Particle Image

Velocimetry and Dynamic Mode

Decomposition (DMD) in a Rectangular Impinging Jet: Vortex Dynamics and Acoustic Generation Fluids 2021, 6, 429. https://doi.org/ $10.3390 /$ fluids 6120429

Academic Editor: Mehrdad Massoud

Received: 10 August 2021

Accepted: 26 October 2021

Published: 27 November 2021

Publisher's Note: MDPI stays neutral with regard to jurisdictional claims in published maps and institutional affiliations.

Copyright: (c) 2021 by the authors. Licensee MDPI, Basel, Switzerland. This article is an open access article distributed under the terms and conditions of the Creative Commons Attribution (CC BY) license (https:/ / creativecommons.org/licenses/by/ $4.0 /)$.
1 Faculty of Engineering, Beirut Arab University, Beirut P.O. Box 11-5020, Lebanon; h.assoum@bau.edu.lb

2 School of Engineering, Lebanese American University, Byblos P.O. Box 36, Lebanon; jana.hamdi@lau.edu.lb 3 Laboratoire des Sciences de l'Ingénieur pour l'Environnement (LaSIE UMR CNRS 7356), La Rochelle Université, 17042 La Rochelle, France; marwan.alkheir@univ-lr.fr (M.A.); kamel.abed-meraim@univ-lr.fr (K.A.M.); asakout@univ-lr.fr (A.S.)

4 LHEEA UMR CNRS 6598, Centrale Nantes, 44321 Nantes, France; hassan.assoum@gmail.com

5 Mechanical Engineering Department, Prince Mohammad Bin Fahd University, Al Khobar 34754, Saudi Arabia

* Correspondence: melhassan@pmu.edu.sa

\begin{abstract}
Impinging jets are encountered in ventilation systems and many other industrial applications. Their flows are three-dimensional, time-dependent, and turbulent. These jets can generate a high level of noise and often present a source of discomfort in closed areas. In order to reduce and control such mechanisms, one should investigate the flow dynamics that generate the acoustic field. The purpose of this study is to investigate the flow dynamics and, more specifically, the coherent structures involved in the acoustic generation of these jets. Model reduction techniques are commonly used to study the underlying mechanisms by decomposing the flow into coherent structures. The dynamic mode decomposition (DMD) is an equation-free method that relies only on the system's data taken either through experiments or through numerical simulations. In this paper, the DMD technique is applied, and the spatial modes and their frequencies are presented. The temporal content of the DMD's modes is then correlated with the acoustic signal. The flow is generated by a rectangular jet impinging on a slotted plate (for a Reynolds number $R e=4458$ ) and its kinematic field is obtained via the tomographic particle image velocimetry technique (TPIV). The findings of this research highlight the coherent structures signature in the DMD's spectral content and show the cross correlations between the DMD's modes and the acoustic field.
\end{abstract}

Keywords: impinging jet; tomographic PIV; DMD; spectral signature; K-H vortices; spatial modes

\section{Introduction}

With the rapid development of technology and science, the quantity of data generated in the fluid mechanic's field, either through numerical simulations or through real experiments, has increased significantly. These huge amounts of data should be analyzed to obtain a better understanding of the processes being studied. However, dealing directly with these data constitutes a bumpy road and cannot even provide the required information with accuracy. Consequently, some methods of approximating a high-dimensional system by a lower-dimensional one, called model reduction techniques, were developed based on the principle that the flow behaves in a low-dimensional manner [1]. These methods provide a simpler way to study the underlying mechanisms by decomposing the flow behavior into coherent structures [2]. Some of these methods are proper orthogonal decomposition (POD) [3-5], balanced proper orthogonal decomposition (BPOD) [6], and dynamic mode decomposition (DMD) [7].

Dynamic mode decomposition, introduced in 2010 by Schmid [7], computes a set of modes associated with eigenvalues, where these DMD modes form spatial patterns 
representing the flow coherent structures, while the eigenvalues are used to indicate the fixed oscillation frequencies connected to these modes and the decay/growth rate [8]. DMD is an equation-free method that relies only on the system's data taken either through experiments or through numerical simulations and provides a linear model representing how these spatial-temporal coherent structures evolve over time.

After its development, many extensions were added to the dynamic mode decomposition method; that is, when dealing with non-linear systems, it is strongly connected to the spectral analysis of the linear infinite dimensional Koopman operator [9,10]. For incomplete measurements of the system's data, compressive sampling extension can be used to extract the DMD eigenvalues (which are equal to those of the complete system) and reconstruct from them the full-state DMD modes [11]. For multi-scale dynamical systems, multi-resolution dynamic mode decomposition mrDMD can be used to separate slow modes (having eigenvalues near the origin) from the faster ones, and so on [12]. For controlled systems, dynamic mode decomposition with control DMDc can provide an accurate input/output model that includes the impact of the control signals (as they corrupt the standard DMD modes and their associated dynamics) [13].

Thanks to its ability to extract spatial-temporal coherent structures from complex flows without referring to any governing equation, DMD was used widely in the fluid domain. It was applied to simulated flows such as the turbulent cavity flow [14], the wake of a high-speed train [15], and the flow around a cylinder cascade [16], as well as to experimental data like the separation of the flow over a blunt plate [17], impinging circular jet [18], confined turbulent jet [19], and so on.

Impinging jets exhibit rich fluid-structure interaction phenomena and tare accompanied in some cases by a self-sustaining/resonating loop. This flow involves an upstream propagating pressure created by the impingement of downstream coherent structures on a surface (Ho and Nosseir $[20,21]$ ). This upstream propagating wave forces the shear layer close to the exit of the jet to oscillate, inducing a collective interaction in which large coherent vortex rings are born. In ventilation systems, these jets become a source of noise and should be controlled. In order to develop new strategies of flow control, one should understand the interaction between the main coherent structures of the flow and the pressure fluctuations generated at the impingement of these latter on a given surface. An example of the main coherent structures in a jet shear layer is the Kelvin-Helmholtz instability, which also plays an important role in the entrainment mechanism in free jets (El Hassan et al. [22,23]).

Thus, this paper aims to investigate the dynamics of the coherent structures involved in a rectangular impinging jet using DMD. The dynamic mode decomposition algorithms are first presented; then, it is applied to the kinematic field of a rectangular jet impinging on a slotted plate obtained via tomographic particle image velocimetry measurements [24-32]. The DMD modes are extracted and presented with their associated spectrum. The shedding frequency of large vortical structures is then extracted from the transversal velocity and compared to that of the DMD's modes [33]. Cross-correlations between the acoustic signal and the DMD modes are presented and analyzed. The conclusions are then summarized, and perspectives are proposed.

\section{Theoretical Background: DMD Method}

The dynamic mode decomposition technique is an equation-free method that extracts coherent flow structures along with their associated temporal dynamics; it can be applied to both numerical simulations and experimental data.

The algorithm of the DMD is presented in Table 1. It starts by taking a series of flow field measurements separated by a constant time interval $\Delta t$, then two measurement matrices $X$ and $X^{\prime}$ can be constructed, where $X^{\prime}$ is a matrix shifted one time step from $X[7,34-36]$. The matrix $X^{\prime}$ can be then set as a function of $X$ by assuming a linear mapping $A$ that relates a given measurements vector to the next one $v_{i+1}: X^{\prime}=A X$. 
Table 1. Dynamic mode decomposition algorithm.

\begin{tabular}{|c|c|}
\hline Data matrices & $\begin{array}{c}X=\left\{x_{1}, x_{2}, \ldots, x_{n-1}\right\} \\
X^{\prime}=\left\{x_{2}, x_{3}, \ldots, x_{n}\right\}\end{array}$ \\
\hline SVD & $X \approx U_{r} \Sigma_{r} V_{r}^{*}$ \\
\hline Building $\widetilde{A}$ & $\widetilde{A}=U^{*} A U=U^{*} X^{\prime} V \Sigma^{-1}$ \\
\hline Eig. decomposition of $\widetilde{A}$ & $\widetilde{A} \omega=\omega \Omega$ \\
\hline DMD modes $\Phi$ & $\Phi=X^{\prime} V \Sigma^{-1} \omega$ \\
\hline System's solution & $x(t) \approx \Phi e^{\check{\Omega} t} b=\sum_{k=1}^{r} \Phi_{k} e^{w_{k} t} b_{k}$ \\
\hline
\end{tabular}

Singular value decomposition (SVD) to the measurement's matrix is then applied. This SVD operation helps in truncating the data to low rank. Then, a similar transform $\widetilde{A}$ of the matrix $A$ can be built by projecting $A$ onto $U$.

The exact dynamic modes $\Phi$ corresponding to the exact eigenvectors of the matrix $A$ are then calculated [37].

The final step in this algorithm is the development of the system's solution, which can be used to predict the flow's state at future times: $x(t) \approx \Phi e^{\check{\Omega} t} b=\sum_{k=1}^{r} \Phi_{k} e^{w_{k} t} b_{k}$ where $w_{k}=\ln \left(\lambda_{k}\right) / \Delta t, \lambda_{k}$ is the kth eigenvalue, and $\check{\Omega}$ is a diagonal matrix having the values $w_{k}$ along its diagonal.

\section{Experimental Setup}

\subsection{Impinging Jet}

The experimental set-up employed for this study is shown in Figure 1. The flow is generated through a compressor (1), which is driven by a variable frequency drive (2). The VFD allows changing the frequency of rotation of the compressor and, therefore, the exit velocity of the flow. The airflow crosses a $1 \mathrm{~m}^{3}$ settling chamber (3) fitted with metal grids to guarantee acoustic insulation of the duct from the blower. Next, it passes through a rectangular tube $1250 \mathrm{~m}$ in length with a $90 \times 190 \mathrm{~mm}^{2}$ cross section (4), which is extended by a rectangular convergent outlet (5). Honeycomb sections are inserted into the rectangular tube to smooth the flow and eliminate any mean swirl. Hence, the set-up creates a free jet $H=10 \mathrm{~mm}$ high and $L z=190 \mathrm{~mm}$ wide. Parallel to the convergent outlet, a $4 \mathrm{~mm}$ thick aluminum plate (6) is fitted with a slot with the same dimensions as the convergent outlet and perfectly aligned with it using a displacement system. The plate is set parallel to the convergent outlet and aligned with the latter. The nozzle to plate distance is denoted by $L(L / H=4$ in this paper). The Reynolds number is calculated as follows: $R e=U_{0} \cdot H / v$, where $v$ is the kinematic viscosity of air at $10^{\circ} \mathrm{C}$, and $U_{0}\left(U_{0}=6.3 \mathrm{~m} / \mathrm{s}\right)$ is the initial velocity at the exit of the jet. The Reynolds number $R e=4458$ was used in this work.

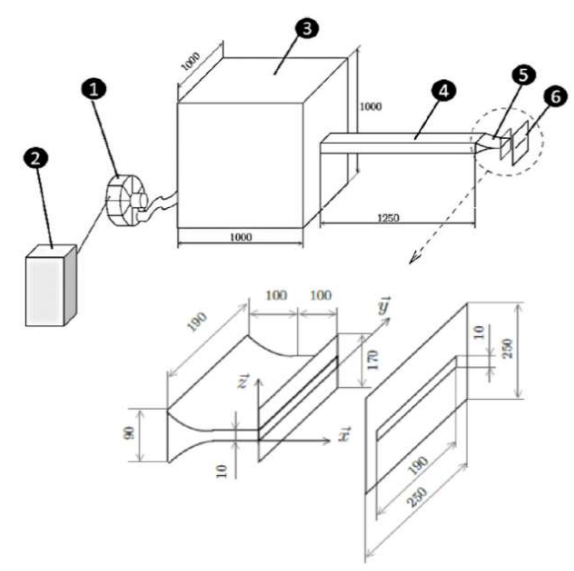

Figure 1. Experimental setup. (1) Compressor, (2) variable frequency drive, (3) settling chamber, (4) duct, (5) rectangular convergent outlet, and (6) slotted plate. 


\subsection{Tomographic PIV}

The velocity field of this study is obtained using a high-speed tomographic particle image velocimetry system (TPIV). This technique takes two consecutive and separate images of the flow (Illuminated by a laser volume) and cross-correlates them to obtain the kinematic field. The interval between the images " $\Delta t$ " is a function of the initial velocity of the jet and the depth of view. High-speed flows were studied using a pulsed laser with a sufficiently small interval. In fact, by looking at a small interval, the displacement caught by the camera is small, providing a high spatial and temporal resolution of the velocity fields obtained [38]. Recordings were taken during one second.

The TPIV set up is composed of a high repetition rate laser: $527 \mathrm{~nm} \mathrm{Nd:} \mathrm{YLF} \mathrm{LDY}$ 300 Litron. The laser provides a power of $30 \mathrm{~mJ}$ per pulse (each pulse has a duration of $0.15 \mu \mathrm{s}$. Thanks to a combination of divergent lenses, a laser volume (whose dimensions are $40 \times 60 \times 10 \mathrm{~mm}^{3}$ ) is generated to illuminate the flow. Three Phantom V711 cameras with $1280 \times 800$ pixels were employed.

Three Phantom V711 cameras with $1280 \times 800$ voxels were used. The cameras were positioned as follows: two cameras on the right side of the jet exit, one above the other. The third camera was placed on the left of the jet at the same level as camera 1 (Figure 2). These three cameras were located at $40 \mathrm{~cm}$ from the measurement volume. The cameras used were set-up at an angle $\theta$ relative to the normal of the laser plane, which was chosen to optimize the quantity of light received. Moreover, they were provided by a Scheimpflug arrangement, allowing an optimization of the sharpness of the particles. The pulsed laser light has a frequency response of $10 \mathrm{kHz}$. Particle seeding was performed using a TOPAS SLG 270 condenser aerosol generator, which ensures the generation of $4 \mu \mathrm{m}$ particles. For the present measurement, the pressure was set at 4 bars, the temperature of the saturator was $240{ }^{\circ} \mathrm{C}$, and that of the reheater was $300^{\circ} \mathrm{C}$. It should be noted that particle size was measured using the PAM 510 granulometer.

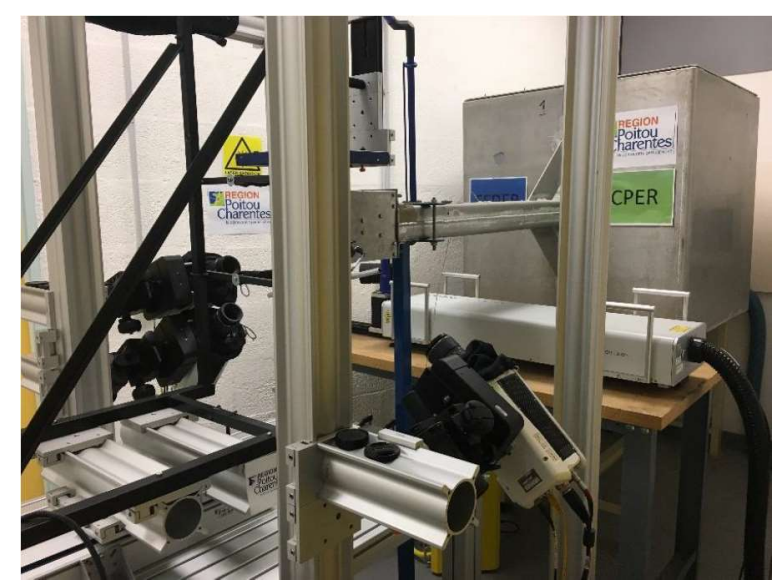

Figure 2. Positions of the cameras.

Couples of PIV images were acquired with a sampling frequency of $2 \mathrm{kHz}$, which is sufficient to capture dominant frequencies standing below $1 \mathrm{kHz}$. Once the particle density was optimized, the time interval $\Delta t$ between the first and the second exposure was adjusted, so that the particles moved a maximum of about 7 to 10 voxels.

The reconstruction of the kinematic field by the algorithm was achieved using the DaVis 8.3.0 software from LaVision, which was used for our measurements. The "calculation of correlation peaks" associated with the "tomographic PIV" module calculates the velocity vector field from the reconstructed volume. The reconstructed image distribution in the volume was analyzed with an iterative multi-grid volume deformation scheme, attaining a 48 -voxel interrogation box size with $75 \%$ overlap, yielding the instantaneous three-dimensional velocity volume. Finally, the post-processing operation of the "tomographic PIV" group was used to reduce the noise in the vector field. 


\section{Results}

\subsection{Reconstruction of the Flow}

The dynamic mode decomposition method aims to represent a data series by orthogonalizing it in time (isolating distinct frequencies). The DMD modes are temporally orthogonal (pure frequencies), but not spatially. The first few DMD modes are illustrated in Figure 3. Note that there are similar modes that come in pairs as each mode has its complex conjugated mode (this is caused by the fact that the imaginary parts of these modes are phase-shifted by $90^{\circ}$ ), and thus only one of them is presented.

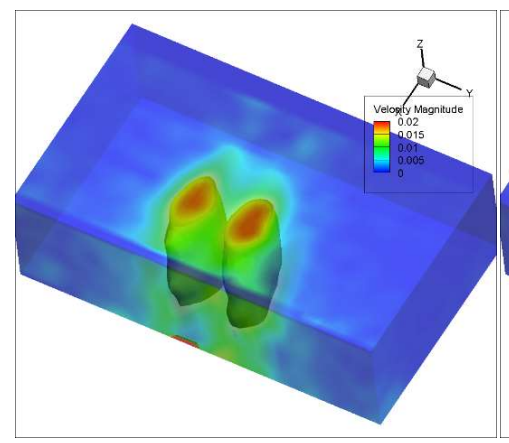

Mode 1

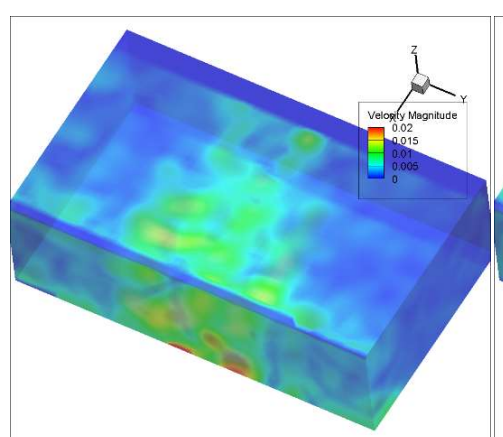

Mode 7

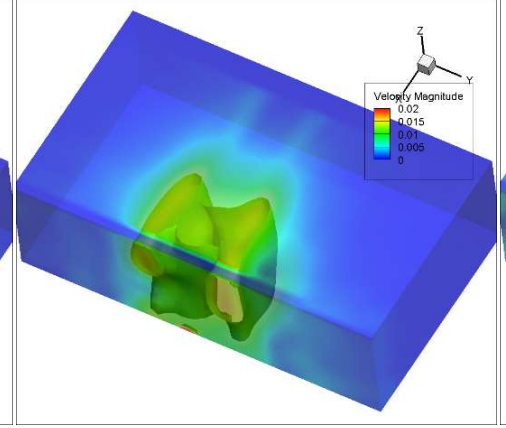

Mode 3

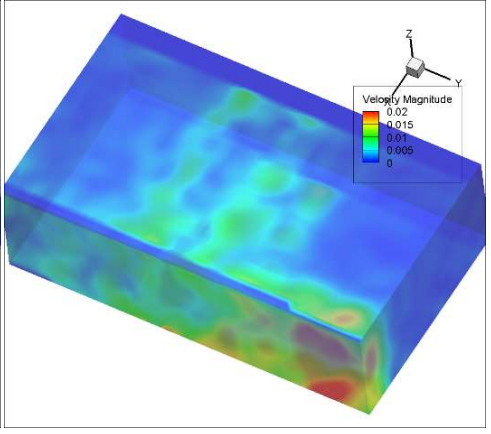

Mode 5

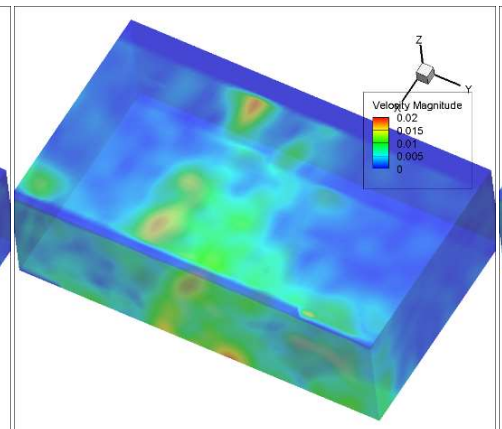

Mode 9

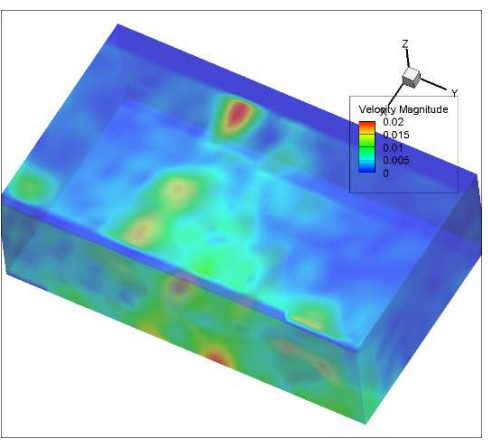

Mode 11

Figure 3. Dynamic mode decomposition modes.

The initial number of the singular values [39] was 1999 ( $n-1$, where $n=2000$ snapshots). Based on the singular value hard threshold algorithm, the first 673 values could be kept, while the remaining could be canceled out as they correspond to noisy data based on this method [40]. This number of modes could be reduced when the kinematic fields are obtained from numerical simulations where the data are much cleaner. In this work, we do not aim to reconstruct the flow. Moreover, to extract and understand the system features, one can simply analyze the number of dynamic modes to reconstruct the flow instead of analyzing the overall system with its noise. Therefore, we use these modes to analyze their spectral contents as shown in the next section.

\subsection{Spectral Content of the DMD Modes}

Lusseyran et al. [33] investigated the coherent structures signature in the spectral content of the DMD's modes. They considered an open cavity flow, where velocity fields were obtained from PIV measurements. Dynamic mode decomposition modes allowed them to identify the spectral signature of centrifugal instability inside the cavity. A similar analysis is used in this section to relate the DMD modes to the energetic large-scale vortical structures in the flow. The transversal velocity field is presented in Figure 4. The temporal velocity profile is extracted at the point $\mathrm{P}(\mathrm{X} / \mathrm{H}=3, \mathrm{Y} / \mathrm{H}=0.6$ and $\mathrm{Z} / \mathrm{H}=0)$, where the passage of the large-scale vortices occurs when travelling from the jet exit towards the impinging wall. Then, the spectral content of the extracted signal is analyzed. 


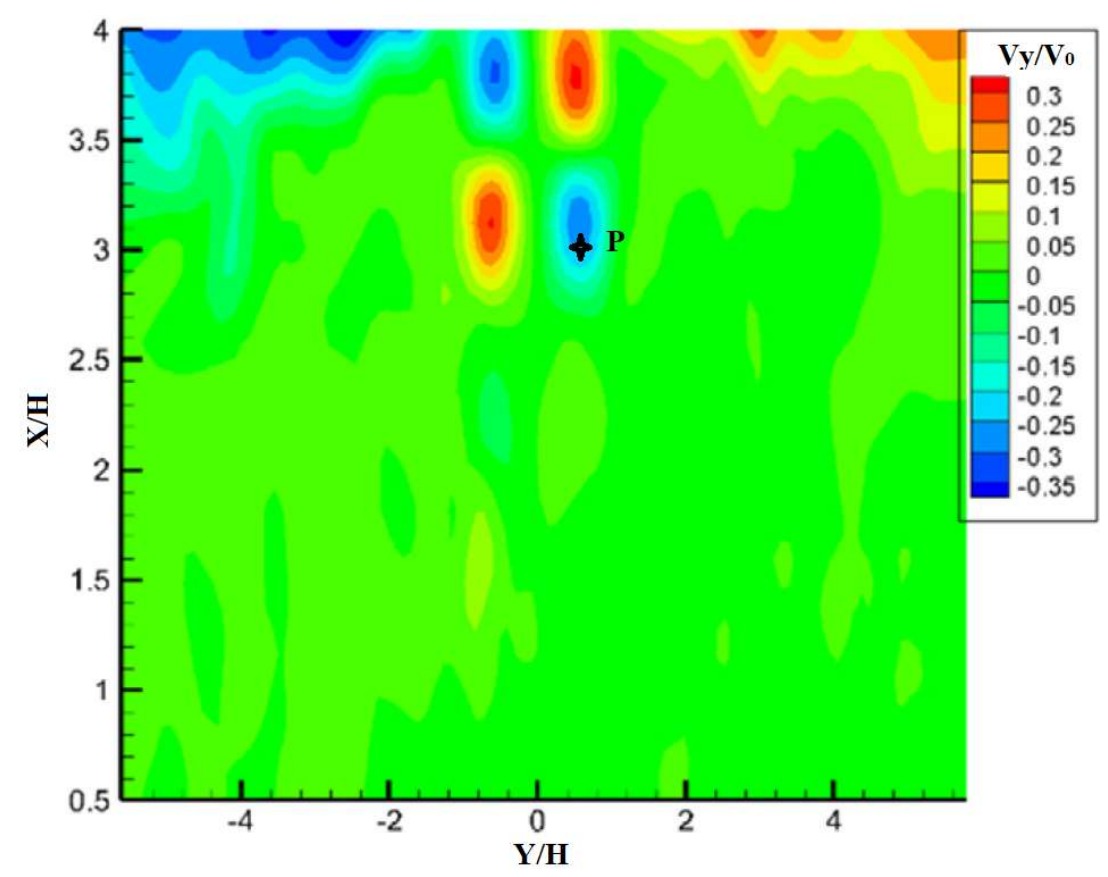

Figure 4. Instantaneous field of the normalized transversal velocity $V_{y} / V_{0}$ at $Z=0$.

The spectrum of the extracted signal $V_{y}$ at the chosen point $(\mathrm{P})$ is shown in Figure 5. One can notice a fundamental peak of frequency at $F_{1}=204 \mathrm{~Hz}$ and a super-harmonic at $2 * F_{1}=408 \mathrm{~Hz}$. The frequency $F_{1}$ corresponds to the frequency of the Kelvin-Helmholtz vortices [25].

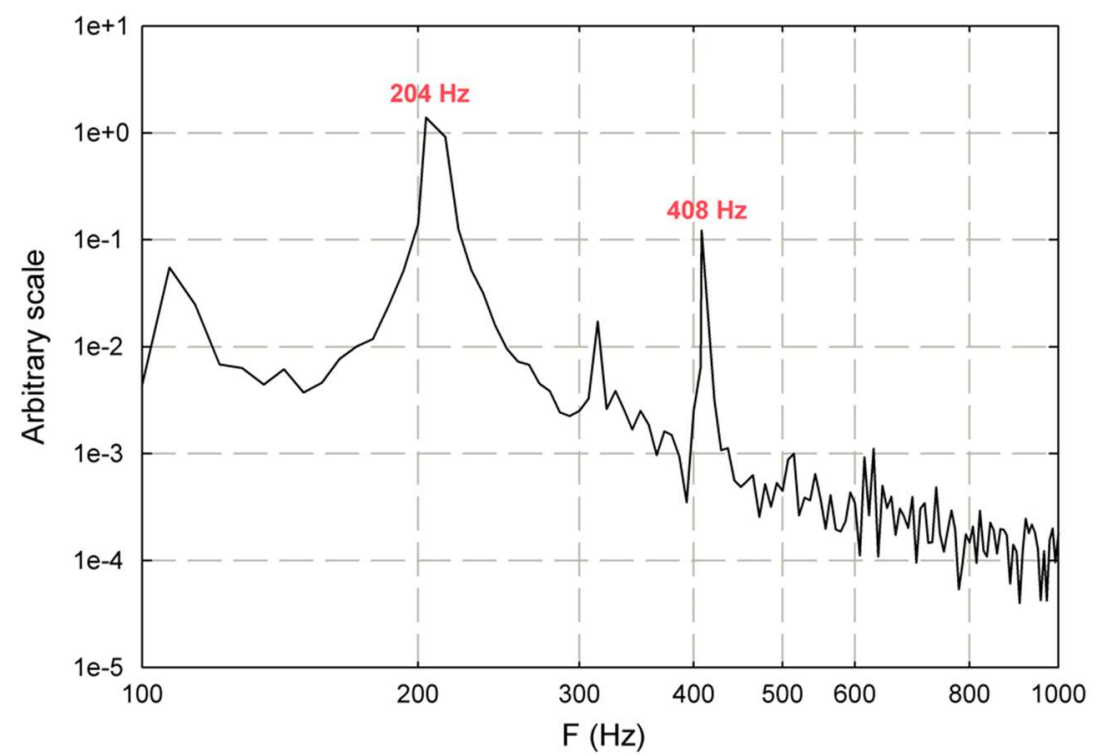

Figure 5. Spectrum of the transversal velocity measured at $\mathrm{X} / \mathrm{H}=3, \mathrm{Y} / \mathrm{H}=0.62$, and $\mathrm{Z} / \mathrm{H}=0$.

In order to observe the signature of the Kelvin-Helmholtz vortices on the DMD's mode, the frequencies of the eleven first DMD's modes are presented in Table 2. Note that each mode and its complex conjugated mode have the same frequency. Thus, only the odd modes are presented in Table 2. 
Table 2. DMD modes and their fundamental frequency.

\begin{tabular}{cc}
\hline DMD Mode & Frequency $\mathbf{( H z )}$ \\
\hline Mode 1 & 402.9 \\
Mode 3 & 200.7 \\
Mode 5 & 200.1 \\
Mode 7 & 610.1 \\
Mode 9 & 219.0 \\
Mode 11 & 220.2 \\
\hline
\end{tabular}

One can note from Table 2 that the first mode (and thus its complex conjugated: mode 2) has almost the same frequency as the super-harmonic present in the spectrum of the transversal velocity $2 * F_{1}$. Modes $3,4,5,6,9,10$, and 11 show the signature of the physical phenomenon of frequency $F_{1}$ in the transversal velocity, which is associated with the passage of the Kelvin-Helmholtz vortices [25,32]. We can note that modes 7 and 8 have a frequency nearly equal to a super-harmonic $3 * F_{1}$. This led us to propose that Kelvin-Helmholtz vortices' frequency $F_{1}$ has an important effect on the DMD, as its spectral signature $\left(F_{1}\right)$ is found in the first DMD's modes, which generally carry the bulk of the energy.

It was shown in [25] that the acoustic signal and the transversal component of velocity have a good correlation and present same peak of frequencies in such configurations. Therefore, in this study, we are interested by the correlation between the acoustic signal and the temporal modes obtained by the DMD. Figures 6 and 7 show the spectrum of the acoustic signal obtained via a microphone placed behind the plate. One can notice almost the same peak in frequency of modes 3 and 5 obtained via the DMD. Therefore, it is of interest to investigate the cross-correlations between the DMD's modes and the acoustic signal.

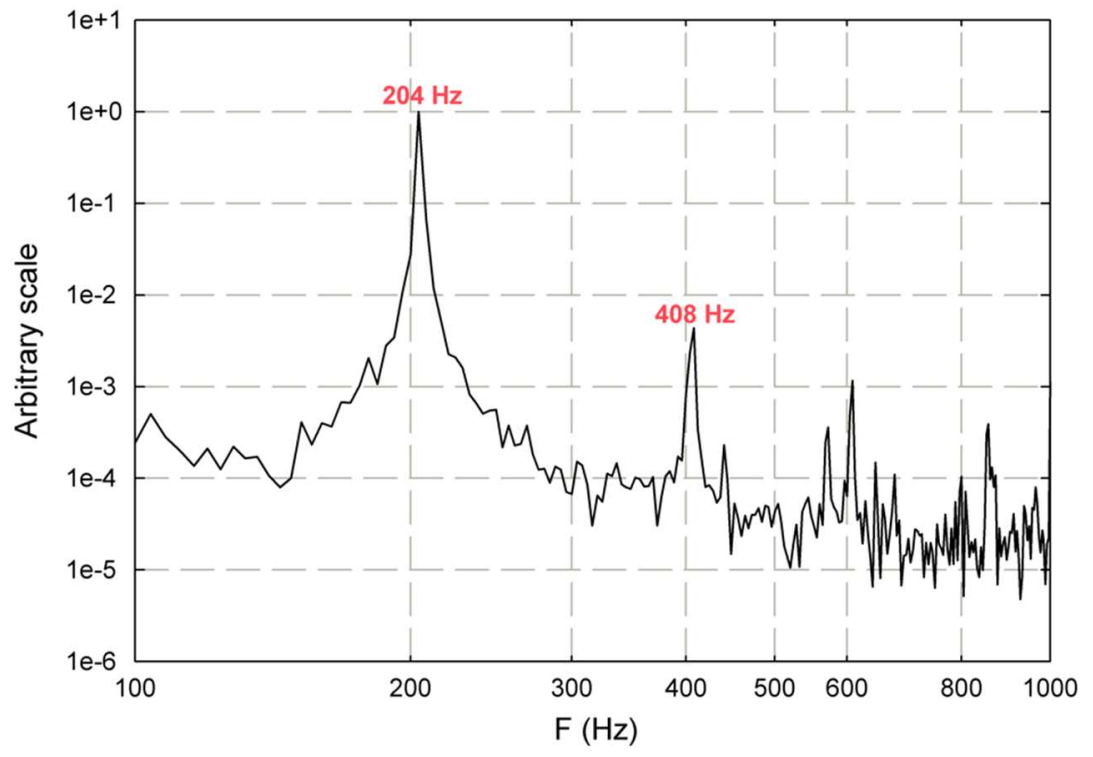

Figure 6. Spectrum of the acoustic signal.

The cross-correlation coefficient $\left(C_{x}\right)$ between the acoustic signal and DMD's modes is presented in Figure 7. It can be seen (Figure $7 \mathrm{~b}$ ) that the cross correlation between the acoustic signal and modes 1 and 7 is very weak $\left(C_{x} \leq 0.1\right)$. Cross correlations between the acoustic signal and modes 5,9 , and 11 have $C_{x} \leq 0.5$. The highest cross-correlation peak $\left(\left|C_{x}\right| \sim 0.9\right)$ is observed (Figure 7a) between the acoustic signal and mode 3. A time delay of $0.8 \mathrm{~ms}$ is obtained for the correlation peak between these two signals. 

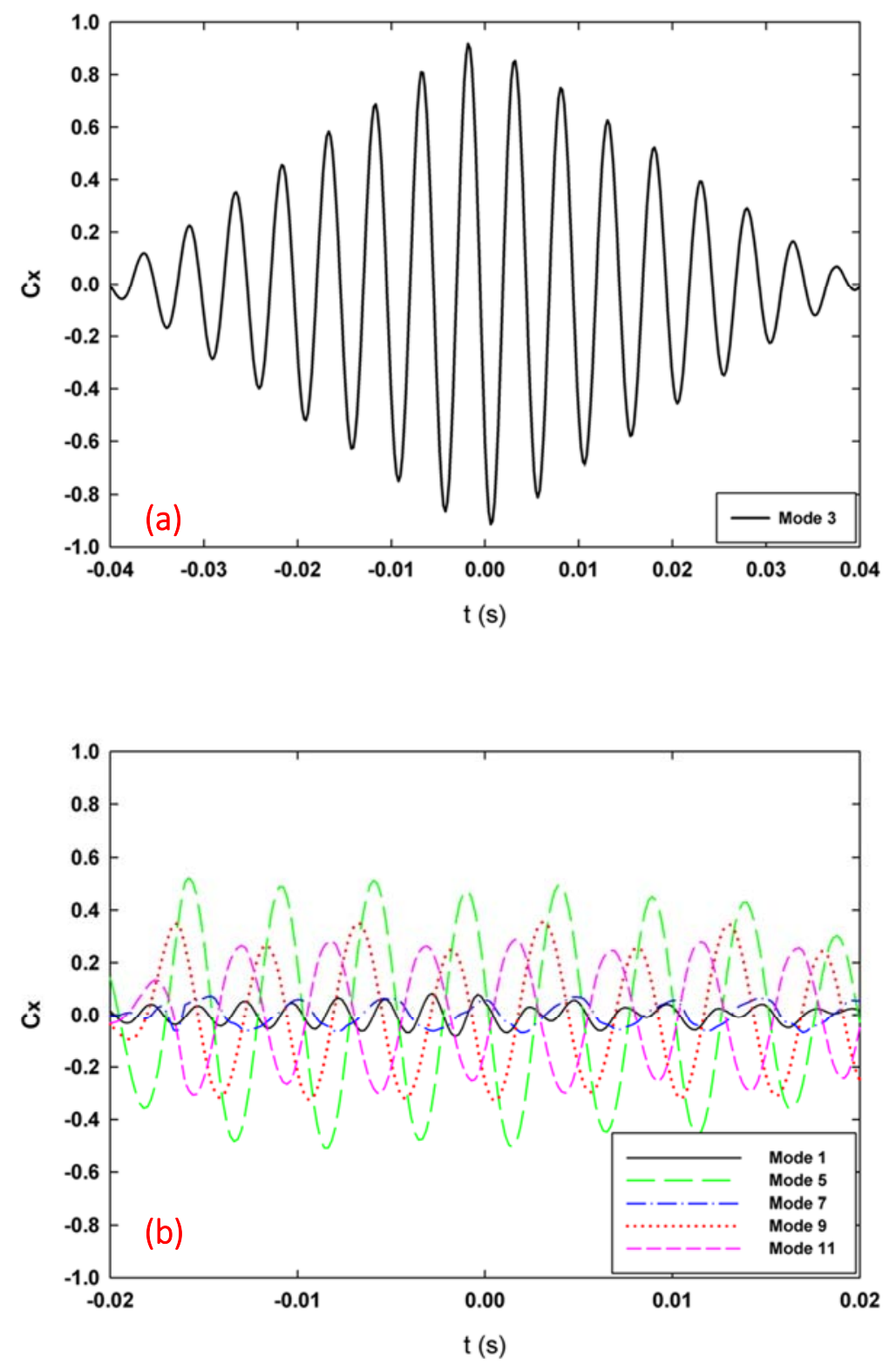

Figure 7. Cross correlation $(\mathrm{Cx})$ between the acoustic signal and the temporal signal of DMD's modes: (a) correlation with DMD mode 3 and (b) correlation with other relevant DMD modes.

One can suggest that the DMD can be used to highlight the important flow dynamics responsible for the acoustic generation in impinging jets. In future work, one could analyze the correlations between the acoustic signal and the kinematic field of the flow reconstructed using the selected modes. Many quantities, such as the turbulent kinetic energy, could be extracted and correlated with the acoustic signal in order to observe their phase evolution, for example, this would allow us to identify the source of energy that feeds the acoustic tones. The importance of such a study would also remain in the identification and isolation of specific dynamic modes that are active in the acoustic generation; this would help in defining control strategies for noise reduction.

\section{Conclusions}

In this work, we investigated the flow dynamics of a rectangular jet impinging on a slotted plate using the TPIV technique and the application of DMD. The three components' velocities of the jet flow are calculated in a volume for a Reynolds number $R e=4458$. The spatial modes obtained from the DMD method were presented. The investigation into the spectral content of the DMD's modes and the transversal velocity shows that the 
Kelvin-Helmholtz vortices' frequency has its signature in the DMD's spectral content. The acoustic spectrum presented similar peaks of that of the transversal velocity. Cross correlations between the acoustic signal and the temporal signal of the DMD modes was calculated. The highest cross-correlation coefficient was obtained between DMD mode 3 and the acoustic signals.

Based on these results, important perspectives could be proposed such as finding a proper criterion of selection of DMD modes that are responsible for the acoustic generation. Moreover, the energy transfers between the aerodynamic (by extracting the TKE from the reconstructed flow) and acoustic fields should be investigated in order to define appropriate control methods that dramatically reduce the noise generation in impinging jets.

Author Contributions: Conceptualization, H.H.A. and M.E.H.; software, H.H.A. and B.O.; investigation, H.H.A. and M.E.H.; resources, A.S., M.A. and K.A.M.; data curation, H.H.A. and J.H.; writing-original draft preparation, H.H.A. and B.O.; writing-review and editing, H.H.A. and M.E.H.; project administration, A.S.; funding acquisition, A.S. All authors have read and agreed to the published version of the manuscript.

Funding: This research received no external funding.

Acknowledgments: The authors wish to thank FEDER and the 'Region of Nouvelle Aquitaine', France for its financial support.

Conflicts of Interest: The authors declare no conflict of interest.

\section{References}

1. Guckenheimer, J. Strange attractors in fluids: Another view. Annu. Rev. Fluid Mech. 1986, 18, 15-29. [CrossRef]

2. Rowley, C.W.; Dawson, S.T. Model reduction for flow analysis and control. Annu. Rev. Fluid Mech. 2017, 49, 387-417. [CrossRef]

3. Sirovich, L. Turbulence and dynamics of coherent structures Part I: Coherent structures. Q. Appl. Math. 1987, 45, 561-571. [CrossRef]

4. Berkooz, G.; Holmes, P.; Lumley, J.L. The proper orthogonal decomposition in the analysis of turbulent flows. Annu. Rev. Fluid Mech. 1993, 25, 539-575. [CrossRef]

5. Rowley, C.W.; Colonius, T.; Murray, R. Model reduction for compressible flows using POD and Galerkin projection. Phys. D Nonlinear Phenom. 2004, 189, 115-129. [CrossRef]

6. Rowley, C. Model reduction for fluids, using balanced proper orthogonal decomposition. Int. J. Bifurc. Chaos 2005, 15, 997-1013. [CrossRef]

7. Schmid, P.J. Dynamic mode decomposition of numerical and experimental data. J. Fluid Mech. 2010, 656, 5-28. [CrossRef]

8. Tu, J.H.; Rowley, C.W.; Luchtenburg, D.M.; Brunton, S.L.; Kutz, J.N. On dynamic mode decomposition: Theory and applications. J. Comput. Dyn. 2014, 1, 391-421. [CrossRef]

9. Mezić, I. Spectral properties of dynamical systems, model reduction and decompositions. Nonlinear Dyn. 2005, 41, 309-325. [CrossRef]

10. Rowley, C.W.; Mezić, I.; Bagheri, S.; Schlatter, P.; Henningson, D.S. Spectral analysis of nonlinear flows. J. Fluid Mech. 2009, 641, 115-127. [CrossRef]

11. Brunton, S.L.; Proctor, J.L.; Tu, J.H.; Kutz, J.N. Compressed sensing and dynamic mode decomposition. J. Comput. Dyn. 2015, 2, 165-191. [CrossRef]

12. Kutz, J.N.; Fu, X.; Brunton, S.L. Multiresolution dynamic mode decomposition. SIAM J. Appl. Dyn. Syst. 2016, 15, 713-735. [CrossRef]

13. Proctor, J.L.; Brunton, S.L.; Kutz, J.N. Dynamic mode decomposition with control. SIAM J. Appl. Dyn. Syst. 2016, 15, $142-161$. [CrossRef]

14. Seena, A.; Sung, H.J. Dynamic mode decomposition of turbulent cavity flows for self-sustained oscillations. Int. J. Heat Fluid Flow 2011, 32, 1098-1110. [CrossRef]

15. Muld, T.W.; Efraimsson, G.; Henningson, D.S. Flow structures around a high-speed train extracted using proper orthogonal decomposition and dynamic mode decomposition. Comput. Fluids 2012, 57, 87-97. [CrossRef]

16. Wang, T. Dynamic mode decomposition on LES result of cylinder cascade wake. In Proceedings of the 5th International Conference of Fluid Flow, Heat and Mass Transfer, Niagara Falls, ON, Canada, 7-9 June 2017.

17. Liu, Y.; Zhang, Q. Dynamic mode decomposition of separated flow over a finite blunt plate: Time-resolved particle image velocimetry measurements. Exp. Fluids 2015, 56, 148. [CrossRef]

18. Meslem, A.; Sobolik, V.; Bode, F.; Sodjavi, K.; Zaouali, Y.; Nastase, I.; Croitoru, C.V. Flow dynamics and mass transfer in impinging circular jet at low Reynolds number. Comparison of convergent and orifice nozzles. Int. J. Heat Mass Transf. 2013, 67, 25-45. [CrossRef] 
19. Semeraro, O.; Bellani, G.; Lundell, F. Analysis of time-resolved PIV measurements of a confined turbulent jet using POD and Koopman modes. Exp. Fluids 2012, 53, 1203-1220. [CrossRef]

20. Ho, C.-M.; Nosseir, N.S. Dynamics of an impinging jet Part 1: The feedback phenomenon. J. Fluid Mech. 1981, 105, 119-142. [CrossRef]

21. Ho, C.-M.; Nosseir, N.S. Large Coherent Structures in an Impinging Turbulent Jet. 1979. Available online: http://adsabs.harvard. edu/abs/1979stsf.procR.7H (accessed on 4 April 2017).

22. Assoum, H.H.; El Hassan, M.; Abed-Meraim, K.; Sakout, A. The vortex dynamics and the self sustained tones in a plane jet impinging on a slotted plate. Eur. J. Mech. B Fluids 2014, 48, 231-235. [CrossRef]

23. El Hassan, M.; Meslem, A. Time-resolved stereoscopic particle image velocimetry investigation of the entrainment in the near field of circular and daisy-shaped orifice jets. Phys. Fluids 2010, 22, 035107. [CrossRef]

24. El Hassan, M.; Meslem, A.; Abed-Meraim, K. Experimental investigation of the flow in the near-field of a cross-shaped orifice jet. Phys. Fluids 2011, 23, 045101. [CrossRef]

25. Assoum, H.H.; El Hassan, M.; Abed-Meraïm, K.; Martinuzzi, R.; Sakout, A. Experimental analysis of the aero-acoustic coupling in a plane impinging jet on a slotted plate. Fluid Dyn. Res. 2013, 45, 045503. [CrossRef]

26. Hamdi, J.; Assoum, H.H.; Abed-Meraïm, K.; Sakout, A. Analysis of the effect of the $3 \mathrm{C}$ kinematic field of a confined impinging jet on a slotted plate by stereoscopic PIV. Eur. J. Mech. B Fluids 2019, 76, 243-258. [CrossRef]

27. Hamdi, J.; Assoum, H.; Abed-Meraïm, K.; Sakout, A. Volume reconstruction of an impinging jet obtained from stereoscopic-PIV data using POD. Eur. J. Mech. B Fluids 2018, 67, 433-445. [CrossRef]

28. Assoum, H.H.; Hamdi, J.; Abed-Meraïm, K.; Al Kheir, M.; Mrach, T.; El Soufi, L.; Sakout, A. Spatio-temporal changes in the turbulent kinetic energy of a rectangular jet impinging on a slotted plate analyzed with High Speed 3D tomographic-particle image velocimetry. Int. J. Heat Technol. 2019, 37, 1071-1079. [CrossRef]

29. Assoum, H.; Hamdi, J.; Abed-Meraim, K.; El Hassan, M.; Ali, M.; Sakout, A. Correlation between the acoustic field and the transverse velocity in a plane impinging jet in the presence of self-sustaining tones. Energy Procedia 2017, 139, 391-397. [CrossRef]

30. Assoum, H.H.; Hamdi, J.; El Hassan, M.; Kamel, A.M.; Kheir, M.E.; Mrach, T.; Asmar, S.E.; Anas, S. Turbulent kinetic energy and self-sustaining tones in an impinging jet using High Speed 3D Tomographic-PIV. Energy Rep. 2020, 6, 6322-6333.

31. El Hassan, M.; Assoum, H.H.; Martinuzzi, R.; Sobolik, V.; Abed-Meraim, K.; Sakout, A. Experimental investigation of the wall shear stress in a circular impinging jet. Phys. Fluids 2013, 25, 077101. [CrossRef]

32. El Hassan, M.; Assoum, H.H.; Sobolik, V.; Vétel, J.; Abed-Meraim, K.; Garon, A.; Sakout, A. Experimental investigation of the wall shear stress and the vortex dynamics in a circular impinging jet. Exp. Fluids 2012, 52, 1475-1489. [CrossRef]

33. Lusseyran, F.; Guéniat, F.; Basley, J.; Douay, C.L.; Pastur, L.R.; Faure, T.M.; Schmid, P.J. Flow coherent structures and frequency signature: Application of the dynamic modes decomposition to open cavity flow. J. Phys. Conf. Ser. 2011, 318, 042036. [CrossRef]

34. Schmid, P.J. Application of the dynamic mode decomposition to experimental data. Exp. Fluids 2011, 50, 1123-1130. [CrossRef]

35. Schmid, P.J.; Meyer, K.E.; Pust, O. Dynamic mode decomposition and proper orthogonal decomposition of flow in a lid-driven cylindrical cavity. In Proceedings of the 8th International Symposium on Particle Image Velocimetry, Melbourne, Australia, 25-28 August 2009.

36. Schmid, P.J.; Li, L.; Juniper, M.; Pust, O. Applications of the dynamic mode decomposition. Theor. Comput. Fluid Dyn. 2010, 25, 249-259. [CrossRef]

37. Kutz, J.N.; Brunton, S.L.; Brunton, B.W.; Proctor, J.L. Dynamic Mode Decomposition: Data-Driven Modeling of Complex Systems; Society for Industrial and Applied Mathematics: Philadelphia, PA, USA, 2016.

38. Elsinga, G.E.; Scarano, F.; Wieneke, B.; van Oudheusden, B.W. Tomographic particle image velocimetry. Exp. Fluids 2006, 41, 933-947. [CrossRef]

39. Sirovich, L.; Kirby, M. Low-dimensional procedure for the characterization of human faces. J. Opt. Soc. Am. A 1987, 4, 519-524. [CrossRef] [PubMed]

40. Gavish, M.; Donoho, D.L. The optimal hard threshold for singular values is $4 / \sqrt{3}$. IEEE Trans. Inf. Theory 2014, 60, 5040-5053. [CrossRef] 